\title{
Prevalence of Gait Features in Healthy Adolescents and Adults
}

\author{
Nak Tscheol Kim*, \\ Seung Jun Moon ${ }^{2 *}$ \\ Moon Seok Park ${ }^{1}$, \\ Kyoung Min Lee ${ }^{1}$, Kug Jin Choi ${ }^{1}$, \\ Woo Young $\mathrm{Choi}^{1}$, Ki Hyuk Sung ${ }^{1}$
}

${ }^{1}$ Department of Orthopedic

Surgery, Seoul National University

Bundang Hospital, Seongnam, Korea,

${ }^{2}$ Department of Orthopedic Surgery,

Korea Workers' Compensation and

Welfare Service Ansan Hospital,

Ansan, Korea

*These authors contributed equally to this work.

Received: January 5, 2021

Revised: February 9, 2021

Accepted: February 18, 2021

\section{Correspondence to}

Ki Hyuk Sung

Department of Orthopedic Surgery, Seoul National University Bundang Hospital, 82 Gumi-ro 173 Beon-gil, Bundang-gu, Sungnam 13620, Korea Tel: +82-31-787-7207

Fax: +82-31-787-4056

E-mail:skh1219@naver.com
Forensic gait analysis is the identification of individuals by their gait style and walking characteristics. This study aimed to examine the prevalence of gait patterns in healthy adolescents and adults. Five hundred healthy participants (mean age, 36.9 years) were enrolled and divided into four age groups: 13-20, $21-35,36-50$, and $\geq 51$ years. The gait of the participants was recorded in a gait analysis laboratory. Five specialists experienced in gait analysis selected several gait features. The prevalence of out-toe, in-toe, planovalgus, and turtleneck was $25.0 \%, 1.6 \%, 6.8 \%$, and $4.2 \%$, respectively. The prevalence of genu varum (10.4\%) was higher than that of genu valgum (5.6\%). Genu valgum and hindfoot valgus were more common in younger than in older subjects $(P=0.018$ and $P=0.029$, respectively). Genu varum was more prevalent in older subjects $(P<0.001)$. The prevalence of out-toe was higher in males $(P<0.001)$, whereas the in-toe and genu valgum were more common in females than in males $(P=0.027$ and $\mathrm{P}=0.038$, respectively). We have documented the prevalence of several gait features in healthy adolescents and adults. These gait features can be used to enhance evidentiary competence in forensic gait analysis and thereby help improve the arrest rate of offenders.

Key Words: Forensic gait analysis; Prevalence; Gait features; Out-toe; Ganu varum

\section{Introduction}

Gait is the movement pattern during locomotion; a key element is its dynamic and repetitive nature [1,2]. Forensic gait analysis is the identification of individuals by gait style and other characteristic features of their walk [3]. It involves the recognition and comparison of gait characteristics to assist in identification. This process generally involves in-depth examination of closed-circuit television (CCTV) footage by a gait specialist for gait patterns and features. Forensic gait analysis was used for the first time in criminal law in the case of R. v. Saunders in The Old Bailey Central Court, London, UK, on July 12, 2000 [4]. Haydn Kelly, a gait specialist, was able to identify the prime suspect as the jewelry thief from CCTV footage. 
Forensic gait analysis has since progressed in several countries. Birch et al. [1] have reported that gait analysts perform well in the comparative identification of suspects from CCTV footage; these individuals can therefore testify as expert witnesses. The authors also developed a tool for assessing the suitability of closedcircuit camera footage for forensic gait analysis [5]. After comparing gait features between CCTV footage and recordings obtained during questioning, it is important to determine their prevalence in the population of interest to establish their distinctiveness. The difference in the prevalence of a gait pattern indicates that the probability of identifying a person differs even if the same individual displays the same gait pattern on CCTV footage recorded at a different location. Relatively little information has been published regarding the prevalence of gait patterns. Therefore, the present study examined the prevalence of gait patterns in healthy Korean adolescents and adults.

\section{Materials and Methods}

\section{Participants}

This prospective cohort study was approved by the institutional review board of our institution. Informed consent was obtained from all the participants or their guardians. The cohort included 500 healthy volunteers, who were divided into the following four age groups: $13-20,21-35,36-50$, and $\geq 51$ years. Gallup Korea enrolled 500 healthy volunteers in Seongnam-si using a stratified sampling method; the group was evenly distributed among age groups and sex. The medical history and lower extremity teleroentgenogram results were obtained for each participant. Participants with any musculoskeletal diseases, any history of orthopedic surgery, or any medical or neurologic condition capable of affecting gait were excluded.

\section{Gait checklist}

A consensus-building session was held by five experienced specialists who perform forensic gait analysis as part of their professional practice; the goal was to select appropriate gait patterns that can be recognized in forensic gait analysis. The panel included individuals from a range of professional backgrounds, including orthopedics and biomechanics. A literature review focusing on gait features was performed and subsequently, several features were selected (Fig. 1): out-toe, in-toe, hindfoot valgus, planovalgus, hindfoot varus, cavus, tip-toe, foot drop, genu valgum, genu varum, genu recurvatum, stiff knee, flexed knee, scissoring, flexed hip gait, Trendelenburg gait, waddling gait, pelvic rotation (external/internal), pelvic obliquity (up/down), pelvic tilt (anterior/posterior), gait symmetry (symmetric/asymmetric), base (wide/narrow), limblength discrepancy, stride length (long/short), ataxic gait, dystonic gait, spastic gait (hemiplegic/diplegic), limping gait, circumduction, turtleneck, lordosis, and kyphosis.

\section{Gait recording and gait analysis}

Gaits were recorded in a dedicated gait analysis laboratory at the Department of Orthopedic Surgery at Seoul National University Bundang Hospital. The participants walked barefoot, with half pants and sleeveless shirts, so that joint movements and trunk motion could be observed. They were asked to walk at a comfortable and usual speed along a straight, 6.5m walkway. Two video cameras (FDR-AX100, Sony Corporation, Beijing, China) were positioned frontally and laterally to the walkway. The frontal camera recorded front and rear walking, and the lateral camera recorded lateral walking. When recording from the front and back, the camera was placed $1.5 \mathrm{~m}$ from the participants in the direction of the gait. When recording laterally, the camera was placed $3 \mathrm{~m}$ away from the gait path and was positioned vertically to the path. The height of the camera was fixed at the middle of the participants. Five trials per participant were recorded. After video recordings were obtained, three-dimensional gait analysis was performed using a motion analysis system (Motion Analysis Corporation, Rohnert Park, CA, USA). This system uses a set of 25 markers attached to specific landmarks of the body. The participants made five or more passes down a $6.5-\mathrm{m}$ walkway, similar to the video recordings. Three-dimensional marker-based data were recorded using 10 infrared digital real-time 
cameras.

Before the main assessments, we performed reliability testing. With a target intraclass correlation coefficient (ICC) value of 0.8 and $95 \%$ confidence interval (CI) width of 0.2 for three examiners, the minimal sample size determined using Bonett's method was 36 patients [6]. Three orthopedic surgeons with 4,5 , and 16 years of experience in independent practice, assessed

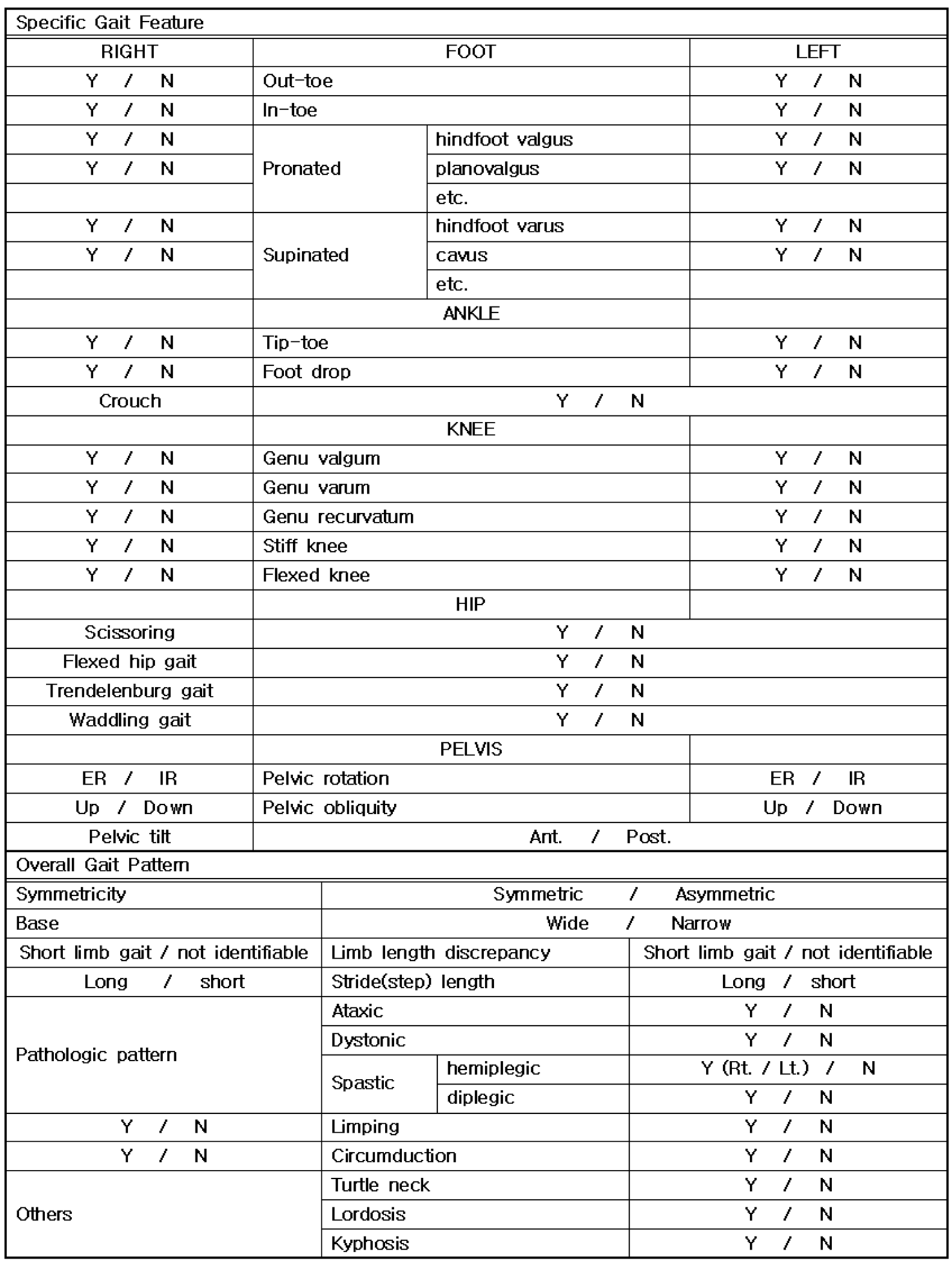

Fig. 1. Checklist of gait features. ER, external; IR, internal. 
recordings in a blinded fashion. Interobserver reliability was determined by ICC and $95 \% \mathrm{Cl}$ in the setting of a two-way mixed-effect model, assuming a single measurement and absolute agreement [7].

After reliability testing, an orthopedic surgeon (K.J.C.) assessed the gaits of 500 participants from the video recordings. No time limit or viewing restrictions were imposed. The assessor could review, slow down, or freeze the video recordings as required. Each gait feature was counted if a deviation was present on one or both sides, and was not counted if a deviation was not present on either side. Each prevalence and $95 \% \mathrm{Cl}$ were calculated using the Poisson approximation to the binomial distribution. The chi-square test was used to group and sex. All statistical analyses were conducted using SPSS software for Windows (version 25.0, IBM Corp., Armonk, NY, USA). All statistical analyses were two-tailed, with P-values of $<0.05$ considered to be statistically significant.

Table 1. Demographic characteristics of the 500 participants

\begin{tabular}{lc}
\hline \multicolumn{1}{c}{ Variable } & Value \\
\hline Age $(\mathrm{yr})$ & $36.9 \pm 16.7$ \\
No. of subject in each age group (male/female) & $256 / 244$ \\
$13-20 \mathrm{yr}$ & $123(64 / 59)$ \\
$21-35 \mathrm{yr}$ & $130(68 / 62)$ \\
$36-50 \mathrm{yr}$ & $125(64 / 61)$ \\
$\geq 51 \mathrm{yr}$ & $122(60 / 62)$ \\
Height $(\mathrm{cm})$ & $165.9 \pm 8.9$ \\
Weight $(\mathrm{kg})$ & $64.2 \pm 12.8$ \\
Body mass index $\left(\mathrm{kg} / \mathrm{cm}^{2}\right)$ & $23.2 \pm 3.5$ \\
\hline Values are presented as mean \pm SD unless otherwise indicated. &
\end{tabular}

Table 2. Interobserver reliabilities for assessing gait features

\begin{tabular}{lcc}
\hline & \multicolumn{2}{c}{ Interobserver reliability } \\
\cline { 2 - 3 } & ICC & $95 \% \mathrm{Cl}$ \\
\hline Out-toe & 0.885 & $0.811-0.935$ \\
In-toe & 0.893 & $0.823-0.940$ \\
Hindfoot valgus & 0.796 & $0.678-0.881$ \\
Planovalgus & 0.761 & $0.629-0.859$ \\
Genu valgum & 0.893 & $0.823-0.940$ \\
Genu varum & 0.932 & $0.886-0.962$ \\
\hline
\end{tabular}

ICC, intraclass correlation coefficient; $\mathrm{Cl}$, confidence interval. analyze the differences in gait features according to age

Table 3. The prevalence of gait feature

\begin{tabular}{|c|c|c|}
\hline Gait feature & Prevalence (\%) & $95 \% \mathrm{Cl}$ \\
\hline \multicolumn{3}{|l|}{ Foot } \\
\hline Out-toe & 25.0 & $21.2-28.8$ \\
\hline In-toe & 1.6 & $0.5-2.7$ \\
\hline Hindfoor valgus & 6.2 & $4.1-8.3$ \\
\hline Planovalgus & 6.8 & $4.6-9.0$ \\
\hline Hindfoot varus & 0.4 & $0.2-1.0$ \\
\hline Cavus & 0.4 & $0.2-1.0$ \\
\hline \multicolumn{3}{|l|}{ Ankle } \\
\hline Tip-toe & 0.0 & - \\
\hline Foot drop & 0.0 & - \\
\hline Crouch & 0.0 & - \\
\hline \multicolumn{3}{|l|}{ Knee } \\
\hline Genu valgum & 5.6 & $3.6-7.6$ \\
\hline Genu varum & 10.4 & $7.7-13.1$ \\
\hline Genu recurvatum & 0.0 & - \\
\hline Stiff knee & 0.0 & - \\
\hline Flexed knee & 0.0 & - \\
\hline \multicolumn{3}{|l|}{ Hip } \\
\hline Scissoring & 0.0 & - \\
\hline Flexed hip gait & 0.0 & - \\
\hline Trendelenburg gait & 0.0 & - \\
\hline Waddling gait & 0.2 & $0.2-0.6$ \\
\hline \multicolumn{3}{|l|}{ Pelvis } \\
\hline Pelvis rotation & 0.4 & $0.2-1.0$ \\
\hline Pelvis obliquity & 0.4 & $0.2-1.0$ \\
\hline Pelvic tilt & 0.0 & - \\
\hline \multicolumn{3}{|l|}{ Overall gait pattern } \\
\hline Asymmetricity & 0.0 & - \\
\hline Base (wide or narrow) & 0.0 & - \\
\hline Limb-length discrepancy & 0.0 & - \\
\hline Stride (step) length (short or long) & 0.0 & - \\
\hline \multicolumn{3}{|l|}{ Pathologic gait } \\
\hline Ataxic gait & 0.0 & - \\
\hline Dystonic gait & 0.0 & - \\
\hline Spastic (hemiplegic/diplegic) & 0.0 & - \\
\hline Limping gait & 0.0 & - \\
\hline Circumduction & 0.2 & $0.2-0.6$ \\
\hline \multicolumn{3}{|l|}{ Other } \\
\hline Turtle neck & 4.2 & $2.7-6.0$ \\
\hline Lordosis & 0.8 & $0.0-1.6$ \\
\hline Kyphosis & 0.0 & - \\
\hline
\end{tabular}

http://www.kjlm.or.kr 
Table 4. Gait features according to age group and sex

\begin{tabular}{|c|c|c|c|c|c|c|c|c|}
\hline \multirow{2}{*}{ Gait feature } & \multicolumn{4}{|c|}{ Age group (yr) } & \multirow{2}{*}{ P-value } & \multicolumn{2}{|c|}{ Sex } & \multirow{2}{*}{ P-value } \\
\hline & $13-20(n=123)$ & $21-35(n=130)$ & $36-50(n=125)$ & $\geq 51(n=122)$ & & Male $(n=256)$ & Female $(n=244)$ & \\
\hline Out-toe & 32 & 25 & 32 & 36 & 0.293 & 97 & 28 & $<0.001$ \\
\hline In-toe & 3 & 3 & 2 & 0 & 0.400 & 1 & 7 & 0.027 \\
\hline Hindfoot valgus & 14 & 8 & 6 & 3 & 0.029 & 16 & 15 & 0.962 \\
\hline Planovalgus & 13 & 9 & 7 & 5 & 0.217 & 20 & 14 & 0.357 \\
\hline Genu valgum & 12 & 10 & 2 & 4 & 0.018 & 9 & 19 & 0.038 \\
\hline Genu varum & 7 & 7 & 14 & 24 & $<0.001$ & 31 & 21 & 0.200 \\
\hline
\end{tabular}

\section{Results}

The mean age and mean body mass index of the 500 study participants were $36.9 \pm 16.7$ years and $23.2 \pm 3.5$ $\mathrm{kg} / \mathrm{m}^{2}$, respectively. Demographic data are presented in Table 1. Interobserver reliabilities for assessing gait features ranged from good to excellent (ICC, 0.7610.932). The highest interobserver reliability was observed for genu varum (ICC, 0.932) and the lowest for planovalgus (ICC, 0.761) (Table 2).

The prevalence of out-toe was $25.0 \%(95 \% \mathrm{Cl}, 21.2-$ $28.8)$, whereas the prevalence of in-toe was $1.6 \%$ (95\% $\mathrm{Cl}, 0.5-2.7)$. Genu varum (10.4\%; $95 \% \mathrm{Cl}, 7.7-13.1)$ was more common than genu valgum $(5.6 \%$; $95 \% \mathrm{Cl}, 3.6-$ 7.6). The prevalence of planovalgus and turtleneck was $6.8 \%(95 \% \mathrm{Cl}, 4.6-9.0)$ and $4.2 \%(95 \% \mathrm{Cl}, 2.7-$ $6.0)$, respectively. The data for other gait features are presented in Table 3.

According to the age, we observed significant differences in the prevalence of hindfoot valgus, genu valgum, and genu varum. Genu valgum and hindfoot valgus were more prevalent in the younger than in older subjects ( $P=0.018$ and $P=0.029$, respectively). The prevalence of genu varum in the older age group was higher in younger subjects $(P<0.001)$. Based on sex, there were significant differences in the prevalence of out-toe, in-toe, genu valgum, and genu varum. The prevalence of out-toe was higher in males $(P<0.001)$, whereas in-toe and genu valgum were more common in female than in males $(P=0.027$ and $P=0.038$, respectively) (Table 4).

Although the genu varum and out-toe were commonly observed, the prevalence of the combination of these two features was low $(3.0 ; 95 \% \mathrm{Cl}, 1.5-4.5)$.
Table 5. The prevalence of combination of gait feature

\begin{tabular}{lcr}
\hline Combination of gait feature & Prevalence (\%) & \multicolumn{1}{c}{$95 \% \mathrm{Cl}$} \\
\hline Out-toe+genu varum & 3.0 & $1.5-4.5$ \\
Planovalgus+genu varum & 0.6 & $0-1.3$ \\
Out-toe+planovalgus & 3.6 & $2.0-5.2$ \\
In-toe+genu valgum & 0.2 & $0.2-0.6$ \\
Planovalgus+genu valgum & 0.8 & $0-1.6$ \\
\hline
\end{tabular}

$\mathrm{Cl}$, confidence interval.

The prevalence of the combination of out-toe and planovalgus was $3.6 \%(95 \% \mathrm{Cl}, 2.0-5.2)$. The prevalence of other combinations of features is presented in Table 5.

\section{Discussion}

Our study assessed the prevalence of gait features that can be used in forensic gait analysis. The most common gait feature was out-toe, followed by genu varum, planovalgus, hindfoot valgus, genu valgum, and turtleneck. Other gait features were observed in less than $1 \%$ of the participants.

The advantage of forensic gait analysis is that, unlike a fingerprint or face, a gait is more difficult to conceal $[8,9]$. Gait evidence is probably not as strong as fingerprint or DNA evidence; however, it can be extremely useful in the absence of conclusive evidence [10]. If the gait features of the suspect and the perpetrator are different, the probability that the suspect is the perpetrator is very low. Contrarily, a similarity between the gait features of the suspect and the perpetrator does not imply that the suspect is the perpetrator. However, if the prevalence of the observed gait feature is very low, the probability that the suspect is the perpetrator increases. Thus, data on the 
prevalence of gait features are important to strengthen evidentiary competence. Our present study is therefore significant, because our results can be used to arrive at a numerical probability.

As mentioned above, the gait specialist Kelly [4] was able to identify the prime suspect as the jewelry thief from CCTV footage. Kelly [4] noted that both the robber and the suspect displayed genu varum. It was determined that no more than $5 \%$ of the relevant UK population exhibit this feature, which was observed in both video of the suspect and video from the crime scene. The prevalence of genu varum is higher in Korea than in the United Kingdom; hence, if gait analysis is performed with this feature in Korea, there is a lower probability of accurate identification. If a gait feature with a relatively high prevalence is identified in recordings of a crime scene, one should consider using combinations of features to enhance accuracy. In the present study, we showed that although the prevalence of genu varum and out-toe was relatively high, the prevalence of the combination of the two features was low. Thus, after analyzing gait features from recordings of both the suspect and the perpetrator, the analyst should identify the most appropriate feature or combination(s) of features.

There are currently 8 million image capturing systems, including 3 million CCTVs and 5 million black boxes, in use in South Korea. As the number of crimes lacking evidence is increasing, CCTV and black box data are being used more frequently as primary investigation tools [11]. With the growing importance of CCTV footage in criminal investigations, forensic gait analysis is becoming increasingly critical to identify criminals. Identification techniques involving gait analysis can be utilized to confirm a person's identity without any contact, similar to fingerprint or iris recognition technologies. Although DiMaggio and Vernon [3] have emphasized the prevalence of gait features in forensic gait analysis, few reports have been published on this aspect to date. Our findings can be used as a reference to evaluate the uniqueness of a specific gait feature.

This study has the following limitation: only Korean individuals were included. Therefore, our results may not be applicable to individuals from other countries. The prevalence of gait features can be changed by differences in cultural habits, such as using a squat toilet, sitting cross-legged, and kneeling on the ground. Studies have shown that hip external rotation, knee flexion, and ankle dorsiflexion are significantly greater in the Asian than in Western populations [12-15]. Additionally, differences in joint movements can affect gait. Therefore, care must be taken when generalizing and applying our results to individuals from other countries.

In conclusion, we determined the prevalence of several gait features in Korean participants that can be used to enhance evidence competence in forensic gait analysis and thereby help improve the positive identification of criminals.

ORCID: Nak Tscheol Kim: https://orcid.org/0000-00016934-8302; Seung Jun Moon: https://orcid.org/00000003-1995-5723; Moon Seok Park: https://orcid.org/00000002-2856-7522; Kyoung Min Lee: https://orcid.org/00000002-2372-7339; Kug Jin Choi: https://orcid.org/00000002-2112-9554; Woo Young Choi: https://orcid.org/00000002-4037-4969; Ki Hyuk Sung: https://orcid.org/00000002-5007-2403

\section{Conflicts of Interest}

No potential conflict of interest relevant to this article was reported.

\section{Acknowledgments}

This research was supported by Projects for Research and Development of Police science and Technology under Center for Research and Development of Police science and Technology and Korean National Police Agency funded by the Ministry of Science, ICT and Future Planning (Grant No. PA-C000001-2015-202).

\section{References}

1. Birch I, Raymond L, Christou A, et al. The identification of individuals by observational gait analysis using closed circuit television footage. Sci Justice 2013;53:339-42.

2. Whittle MW. Gait analysis: an introduction. Oxford: ButterworthHeinemann; 2014.

3. DiMaggio JA, Vernon W. Forensic podiatry: principles and methods. Totowa, NJ: Springer Science \& Business Media; 2011.

4. Kelly H. Old Bailey central criminal court. London: R v Saunders; 2000. 
5. Birch I, Vernon W, Walker J, et al. The development of a tool for assessing the quality of closed circuit camera footage for use in forensic gait analysis.J Forensic Leg Med 2013;20:915-7.

6. Bonett DG. Sample size requirements for estimating intraclass correlations with desired precision. Stat Med 2002;21:1331-5.

7. Lee KM, Lee J, Chung CY, et al. Pitfalls and important issues in testing reliability using intraclass correlation coefficients in orthopaedic research. Clin Orthop Surg 2012;4:149-55.

8. Nixon MS, Carter JN, Shutler JD, et al. New advances in automatic gait recognition. Inform Secur Tech Rep 2002;7:23-35.

9. Ricanek K, Savvides M, Woodard DL, et al. Unconstrained biometric identification: emerging technologies. Computer 2010;43:56-62.

10. Larsen PK, Simonsen EB, Lynnerup N. Gait analysis in forensic medicine.J Forensic Sci 2008;53:1149-53.
11. Cong DN, Khoudour L, Achard C, et al. People re-identification by spectral classification of silhouettes. Signal Process 2010;90:2362-74.

12. Ahlberg A, Moussa M, Al-Nahdi M. On geographical variations in the normal range of joint motion. Clin Orthop Relat Res 1988;(234):229-31.

13. Al-Rawi ZS, Al-Aszawi AJ, Al-Chalabi T. Joint mobility among university students in Iraq. Br J Rheumatol 1985;24:326-31.

14. Kumar S, Sharma R, Gulati D, et al. Normal range of motion of hip and ankle in Indian population. Acta Orthop Traumatol Turc 2011;45:421-4.

15. Hallaceli $\mathrm{H}$, Uruc V, Uysal $\mathrm{HH}$, et al. Normal hip, knee and ankle range of motion in the Turkish population. Acta Orthop Traumatol Turc 2014;48:37-42. 